OPEN ACCESS

Edited by:

Viet-Thanh Pham,

Phenikaa University, Vietnam

Reviewed by:

Shaobo He,

Central South University, China

Sezgin Kaçar,

Sakarya University of Applied

Sciences, Turkey

${ }^{*}$ Correspondence:

Fei Yu

yufeiyfy@@csust.edu.cn

Specialty section:

This article was submitted to

Interdisciplinary Physics,

a section of the journal

Frontiers in Physics

Received: 03 April 2021 Accepted: 14 May 2021

Published: 04 June 2021

Citation:

YuF, Zhang Z, Shen H, Huang Y, CaiS, Jin J and Du S (2021) Design and

FPGA Implementation of a Pseudorandom Number Generator Based on

a Hopfield Neural Network Under

Electromagnetic Radiation.

Front. Phys. 9:690651.

doi: 10.3389/fphy.2021.690651

\section{Design and FPGA Implementation of a Pseudo-random Number Generator Based on a Hopfield Neural Network Under Electromagnetic Radiation}

\author{
Fei Yu ${ }^{1,2 *}$, Zinan Zhang ${ }^{1}$, Hui Shen ${ }^{1}$, Yuanyuan Huang ${ }^{1}$, Shuo Cai ${ }^{1}$, Jie $\mathrm{Jin}^{3,4}$ and Sichun $D u^{5}$ \\ ${ }^{1}$ School of Computer and Communication Engineering, Changsha University of Science and Technology, Changsha, China, \\ ${ }^{2}$ Guangxi Key Laboratory of Cryptography and Information Security, Guilin University of Electronic Technology, Guilin, China, \\ ${ }^{3}$ School of Information and Electrical Engineering, Hunan University of Science and Technology, Xiangtan, China, ${ }^{4}$ College of \\ Information Science and Engineering, Jishou University, Jishou, China, ${ }^{5}$ College of Computer Science and Electronic Engineering, \\ Hunan University, Changsha, China
}

When implementing a pseudo-random number generator (PRNG) for neural network chaosbased systems on FPGAs, chaotic degradation caused by numerical accuracy constraints can have a dramatic impact on the performance of the PRNG. To suppress this degradation, a PRNG with a feedback controller based on a Hopfield neural network chaotic oscillator is proposed, in which a neuron is exposed to electromagnetic radiation. We choose the magnetic flux across the cell membrane of the neuron as a feedback condition of the feedback controller to disturb other neurons, thus avoiding periodicity. The proposed PRNG is modeled and simulated on Vivado 2018.3 software and implemented and synthesized by the FPGA device ZYNQ-XC7Z020 on Xilinx using Verilog HDL code. As the basic entropy source, the Hopfield neural network with one neuron exposed to electromagnetic radiation has been implemented on the FPGA using the high precision 32-bit Runge Kutta fourth-order method (RK4) algorithm from the IEEE 754-1985 floating point standard. The postprocessing module consists of 32 registers and 15 XOR comparators. The binary data generated by the scheme was tested and analyzed using the NIST 800.22 statistical test suite. The results show that it has high security and randomness. Finally, an image encryption and decryption system based on PRNG is designed and implemented on FPGA. The feasibility of the system is proved by simulation and security analysis.

Keywords: PRNG, hopfield neural network, electromagnetic radiation, chaotic degradation, FPGA, security analysis, image encryption and decryption system

\section{INTRODUCTION}

With the rapid development of digital communication technology, especially in today's increasingly popular smart phones and network communication, more and more people are demanding security for private information [1-5]. Cryptography has been commonly used for fast transmission of information and data and can meet the privacy information security requirements. The secure transmission of information and data relies on the randomness of the security keys of information security systems [6-10]. Therefore, the use of high-quality random sequences as security keys and encrypted data is increasingly common in today's information security systems. In the field of information security, pseudo-random number generators (PRNGs), an important part of stream ciphers, can efficiently 
generate random sequences with high randomness and sensitivity and improve the security of information security systems [11, 12]. At the same time, the development of neural network chaotic systems also provides new theoretical basis and ideas for the design of PRNGs, but some information security problems that may arise are of increasing concern. Thus, the construction of high-performance PRNGs using neural network chaotic systems has been taken as an important topic in the research of information security field.

Chaos is unpredictable, irreducible, and sensitive to initial conditions. In recent years, various constructions of chaotic systems have been proposed [13-18]. So far, chaotic systems have been widely used in various fields that already exist, such as synchronization [19-21], secure communication [22, 23], neural network chaotic systems [24-32], and PRNGs [33-38]. Among them, PRNGs based on chaotic systems are the most fundamental applications of chaotic systems. However, the performance of RPNG is greatly affected by the chaotic degradation caused by the computational accuracy [39-44]. To overcome this problem, Chen et al. [39] proposed a PRNG with three different dimensions of quadratic memory hyperchaotic systems as multiple entropy sources, and the XOR operation was performed on the three different dimensions of the entropy sources, and the generated high-quality random sequences passed the ENT and NIST 800-22 tests. Zhao et al. [40] designed a new hyperchaotic system based on a self-turbulent PRNG, where a feedback controller in the system is used to achieve perturbation of other dimensions, thus avoiding the transient cycle phenomenon and, ultimately, overcoming the chaotic degeneracy arising from computational accuracy problems. As far as the techniques of $[39,40]$ are concerned, these two PRNGs are implemented in software using algorithms based on multi-chaotic systems and feedback controllers for self-perturbation of other dimensions, respectively, to overcome the chaotic degeneracy. However, hardware-based implementations do not guarantee flexible use. All the above mentioned are applications of PRNG based on chaotic systems, while the applications of PRNG based on neural networks have been reported rarely.

In recent years, neural network chaotic dynamics has been extensively studied [45-49]. Therefore, PRNGs based on neural network chaotic systems implemented by FPGAs have attracted the attention of more and more researchers. Dong et al [50] controlled the input and output of the six-dimensional cellular neural network generated by each iteration and performed XOR operations on the random sequences generated by the logic mapping. Ultimately, the period of the output sequence can be extended to improve the transient cycle phenomenon and the randomness and unpredictability of the generated random sequence, and the experimental results passed the NIST statistical test. In [36], a novel chaos-based PRNG was designed using an artificial neural network (ANN)-based 2D chaotic oscillator and a ring oscillator structure. VHDL coding was used to synthesize the chip using the XILINX-ISE design tool, and the generated random sequences passed the NIST-800-22 randomness test, proving that the new FPGA-based value of the existence of PRNGs. In [37], a hardware-oriented Chaotic Boltzmann Machines (CBMs) algorithm, which includes fixed-point and shift operations to reduce the hardware resource utilization of the circuit, is proposed. Thus, CBMs are implemented on FPGA, and the computational speed of the FPGA-implemented CBMs is compared with that of the software-implemented CBMs, proving that the FPGA implementation of CBMs outperforms the other solutions. As far as the hardware implementation is concerned, FPGA use already established logic modules and reprogrammable wiring resources to implement the required hardware functions [50]. At the same time, FPGA can take full advantage of hardware parallelism, enabling more tasks to be performed in a fixed cycle as opposed to sequential execution, reducing design and test cycle costs and increasing the diversity and flexibility of PRNGs based on neural network chaotic systems $[37,38]$. But so far, there is no research on PRNG of chaos system based on FPGA Hopfield neural network.

In this work, in order to reduce the impact of chaotic degradation caused by FPGA implementation on the quality of random sequence generation and to improve the randomness of random numbers generated by PRNG, a PRNG with a feedback controller based on Hopfield neural network oscillator containing a feedback controller is designed in this paper. Among them, a feedback controller is used to reduce the chaotic degradation phenomenon and improve the quality of random sequences. First, the dynamical behavior of the threeneuron Hopfield neural network system with one neuron exposed to electromagnetic radiation was analyzed, and the neural network model was implemented using FPGA, and the experimental results were consistent with the software simulation results. Second, a PRNG with a feedback controller based on Hopfield neural network oscillator containing a feedback controller was designed to post-process the generated random numbers to generate high-quality random sequences, and compared with PRNG based on Hopfield neural network chaotic oscillator, the randomness and security of random sequence are analyzed. Finally, a PRNG-based image encryption and decryption system was implemented on FPGA, and passed the simulation and security analysis on matlab platform.

The rest of this paper is presented as follows. In Section 2, the mathematical model of the Hopfield neural network chaotic system is analyzed, simulation results of the FPGA-based model are given, and the FPGA implementation is used. In Section 3, the flow structure of the PRNG proposed in this paper is introduced, the PRNG is implemented on FPGA, and the FPGA-based experimental results with chip statistics are out. In Section 4, the randomness and security of the random sequences generated by the PRNG are analyzed. In Section 5, the flow of implementing PRNG-based image encryption and decryption system on FPGA is presented, and the engineering application results are shown. Finally, Section 6 concludes the paper.

\section{SYSTEM INTRODUCTION AND IMPLEMENTATION BASED ON FPGA}

\subsection{The System Model and Dynamic Analysis of Hopfield Neural Network \\ 2.1.1 The State Model of the System}

More and more attention has been paid to the research of multidimensional chaos systems based on Hopfield neural networks. Many different chaotic systems based on Hopfield neural networks have been successfully introduced, such as [24,29]. Recently, Lin et al. [29] proposed a three-neuron Hopfield neural network system with one neuron exposed to electromagnetic radiation. Under different control parameters, the system shows rich chaotic dynamic behavior, and the corresponding lyapunov exponents simulation results further verify this result. This system is given by the following equation 


$$
\left\{\begin{array}{l}
\dot{x}=-x-a \tanh (y)+b \tanh (z)+c x Q(w) \\
\dot{y}=-y+d \tanh (x)+e \tanh (y)-f \tanh (z) \\
\dot{z}=-z+g \tanh (x)+h \tanh (y) \\
\dot{w}=m x-n w \\
Q(w)=\alpha-\beta|w|
\end{array}\right.
$$

In Eq. 1, $x, y, z$ and $w$ are system variables, where $x, y$, and $z$ represent the membrane voltages of three neurons respectively, $x$ describes the magnetic flux of neurons exposed to electromagnetic radiation, and $\tanh (i)$ is a hyperbolic tangent function, which is the neuron activation function and represents the input voltage of neuron $i=(x, y, z, w) . Q(w)$ is memory conductance of a flux-controlled memristor, which represents the coupling relationship between the coupling of magnetic flux and membrane potential in neurons exposed to electromagnetic radiation. $\alpha$ and $\beta$ are two electromagnetic radiation parameters. Therefore, Eq. 1 represents the state equation of three neuron Hopfield neural network, where neuron $x$ is exposed to electromagnetic radiation.

\subsubsection{Discrete Model of System}

The discretization process of the neural network model is the key to realize the neural network model on FPGA. According to the equation obtained after the discrete processing, we can determine the number of registers, adders, subtractors, multipliers, comparators, and other modules needed in the hardware implementation. In this study, the whole discretization process is accomplished by RK4 numerical algorithm. The mathematical equation of this numerical algorithm is given by Eq. 3 .

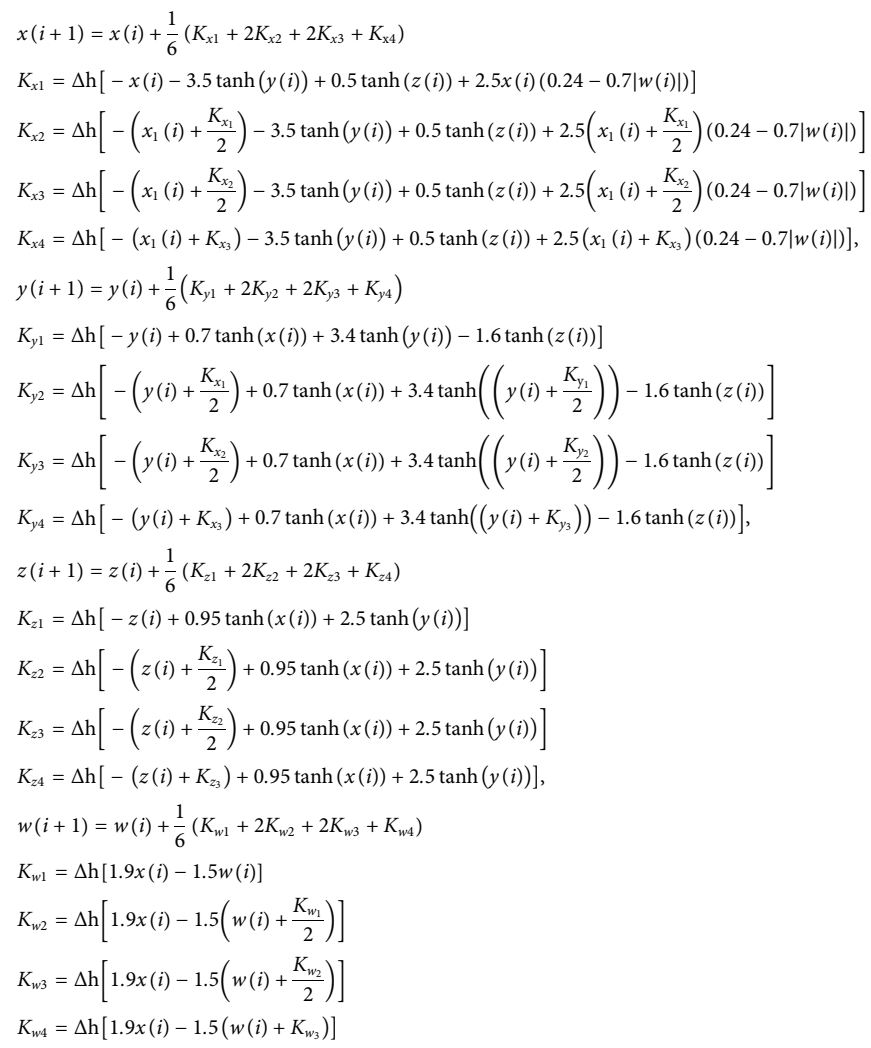

where the step size of each iteration is $\Delta h=0.001$, and $K_{j 1}, K_{j 2}, K_{j 3}, K_{j 4}(j=x, y, z, w)$ respectively represent the slopes of four points in one iteration. In an iterative process, $(x(i), y(i), z(i), w(i))$ provide data for the system, while $(x(i+1), y(i+1), z(i+1), w(i+1))$ obtain data to provide data for the next iteration.

In Eq. 1, the hyperbolic tangent function, which is superior to the sigmoid activation function, is taken as the neuron activation function. Look-up table has always been a traditional way to realize the hyperbolic tangent function, but its implementation on FPGA is very challenging due to the limitation of hardware quantity. Kwan et al. [49] introduced a simple sigmoid-like second-order piecewise activation function, which can be implemented directly in hardware and is close to hyperbolic tangent function. Therefore, the tanh-like bipolar function and the simple sigmoid-like secondorder piecewise function are given by

$$
\begin{gathered}
\tanh (i)=G_{s}(i)=\left\{\begin{array}{l}
1, L \leq i \\
H_{s}(i),-L<i<L \\
-1, i \leq-L
\end{array}\right. \\
H_{s}(i)=\left\{\begin{array}{l}
i(\mu-\theta i), 0 \leq i<L \\
i(\mu+\theta i),-L<i<0
\end{array}\right.
\end{gathered}
$$

Here $\mu=1$ and $\theta=0.25$ represent the slope and gain of $H_{s}(i)$, and $L=2$ determines the length of the middle area. Eq. 3, Eq. 4 and Eq. 5 provide a guarantee for the implementation of neural network system model on FPGA with RK4 numerical algorithm.

\subsubsection{Dynamic Analysis of the System}

In this paper, because the ODE45 function on the Matlab platform is basically the same as the RK4 algorithm in the discrete process, the dynamics analysis of the neural network chaotic system is completed on the Matlab platform on the basis of the ODE45 function and Eq. 4.

When the system parameters are set as $a=3.5, b=0.5, c=$ $2.5, d=0.7, e=3.4, f=1.6, g=0.95, h=2.5, m=1.9, n=1.5, \alpha=$ $0.24, \beta=0.7$ and the initial condition is selected as $(0.1,0.1,0.1,0.1)$. The step size of each iteration is $\Delta h=0.001$. The Lyapunov exponential spectrum of Eq. 1 with respect to parameter $a$, as shown in Figure 1. It is clear from the Figure $1 \mathrm{~A}$ that there is a positive Lyapunov exponent in the system and that when $a=3.5$, the Lyapunov exponent is $L E_{1}=0.324, L E_{2}=0.009, L E_{3}=-0.378, L E_{4}=-1.675$. It can be seen from Figure 1B that on the lyapunov exponent, $L E_{1}$ is greater than $0, L E_{2}$ is equal to $0, L E_{3}$ and $L E_{4}$ are less than 0 . When a dynamical system obtains a correct initial condition, the orbit of the system tends to a certain steady state as time passes. This special steady-state is called the attractor of the system. In this paper, the phase diagram of the system is simulated by Matlab which is shown in Figure 2. Figure 3 shows the time series of state variables $x$ and $w$. In order to further explore the dynamic behavior of neural network chaotic system, when the parameters of neural network chaotic system remain unchanged, the attractor basin of its initial state change is considered to verify the multi stability of the system. Setting the initial state of $x$ and $z$ as 0 , we can draw the attractors of $y$ and $w$ initial plane, in which red, blue and yellow represent different types, different structures and different amplitudes of attractors. 


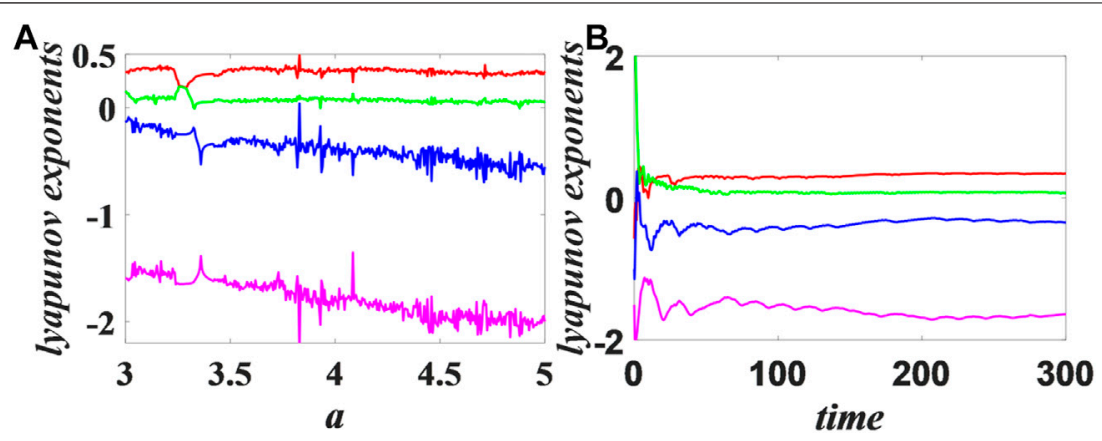

FIGURE 1 | Eq. 1 sets the parameters as $a=3.5, b=0.5, c=2.5, d=0.7, e=3.4, f=1.6, g=0.95, h=2.5, m=1.9, n=1.5, \alpha=0.24, \beta=0.7$ and the initial condition is selected as $(0.1,0.1,0.1,0.1)$. (A) is the spectrum of Lyapunov exponent with parameter a $\in(3,5)$, (B) is the spectrum of Lyapunov exponent with time.
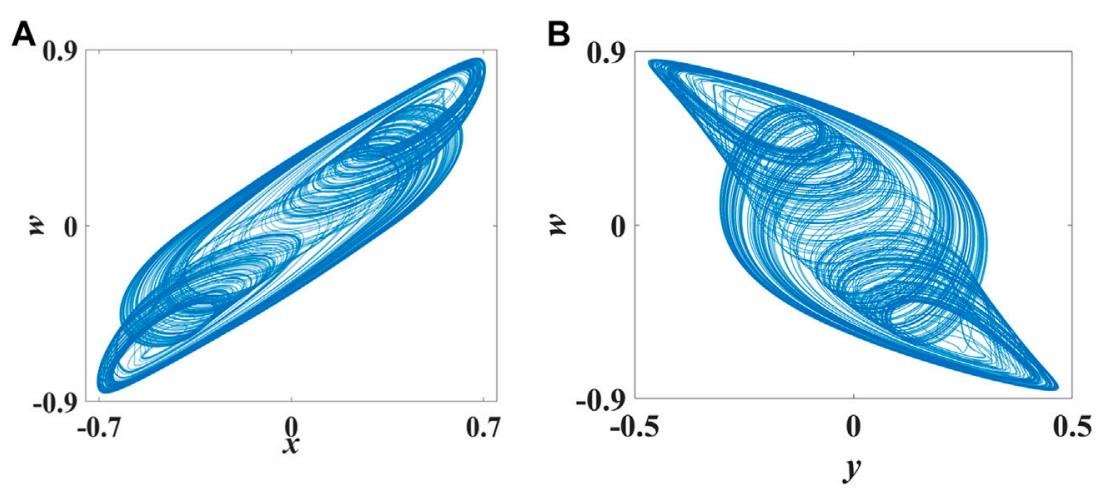

FIGURE 2 | Phase diagram of the Hopfield neural network chaotic system. (A)

A) $x-w$ plane phase diagram; (B) $y-w$ plane phase diagram.

Similarly, the basins of attraction of the initial planes $x$ and $z$ can be drawn in the graph, as shown in Figure 4. Therefore, the Hopfield neural network chaotic system has rich dynamic behavior.

\subsection{Implementation of Hopfield Neural Network System on FPGA}

In this paper, a three-neuron Hopfield neural network system with neuron $x$ exposed to electromagnetic radiation is implemented on the Vivado 2018.3 design platform, and the required modules, such as adders, subtractors, multipliers and comparators, are obtained or created using the IP-CORE generator developed for the platform. The source files of the RK4 numerical algorithm and Eq. 1 are constructed using the required modules and Verilog HDL hardware language under IEEE 754-1985 high precision 32-bit floating point standard. The flow block diagram of the FPGA-based neural network chaotic oscillator has been shown in Figure 5.

As shown in Figure 5, the Hopfield neural network chaotic oscillator has four input signals and five output signals. Start and Clk are 1-bit input signals, which are used to synchronize each module unit. $\Delta h$ and the initial value $\left(X_{0}, Y_{0}, Z_{0}, W_{0}\right)$ are both 32-bit input signals, where $\Delta h=0.001$ is a parameter representing the step size. These two input signals are obtained from the outside and can be easily modified. The four 32-bit output signals (X_Out, Y_Out, Z_Out, W_Out) are input to the floating to fixed unit and used as the initial values of the next iteration of the Hopfield neural network chaotic oscillator. The floating to fixed unit converts the input 32-bit floating point number into 14-bit fixed-point number. The DAC unit converts the output signal of the floating to fixed unit into an analog signal, and then outputs the analog signal $(x, w)$ to Xilinx ZYNQ-XC7Z020 chip. The validity of the output signals $(x, w)$ is determined by the $X Y Z W_{-}$Ready signal of 1-bit. The simulation result of vivado simulator are shown in Figure 6. Then, the Xilinx ZYNQ-XC7Z020 chip is connected to the computer and oscilloscope respectively, and the bitstream file generated by vivado2018.3 platform is transmitted to the chip. The result of oscilloscope is shown in Figure 7. The results show that the phase diagram of the Hopfield neural network with neuron $x$ exposed to electromagnetic radiation based on FPGA is consistent with its MATLAB simulation phase diagram, which verifies the validity of the FPGA.

\section{DESIGN AND FPGA IMPLEMENTATION OF PRNG}

\subsection{Design of PRNG}

In this part, PRNG based on three-neuron Hopfield neural network with neuron $x$ exposed to electromagnetic radiation is composed of entropy source, feedback controller unit, sampling quantization unit and post-processing unit. The structure of PRNG is shown in 
A

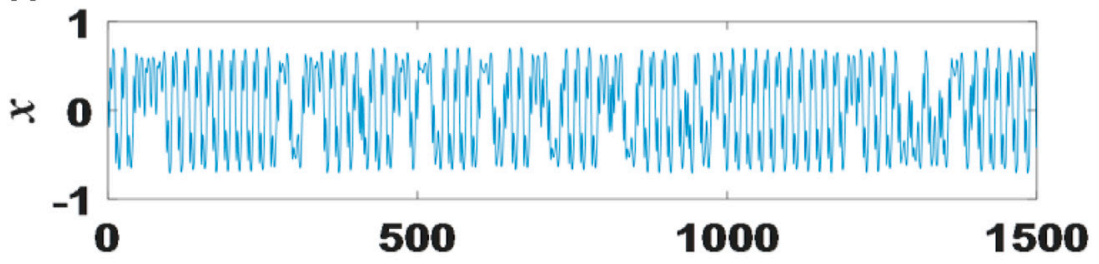

B

t

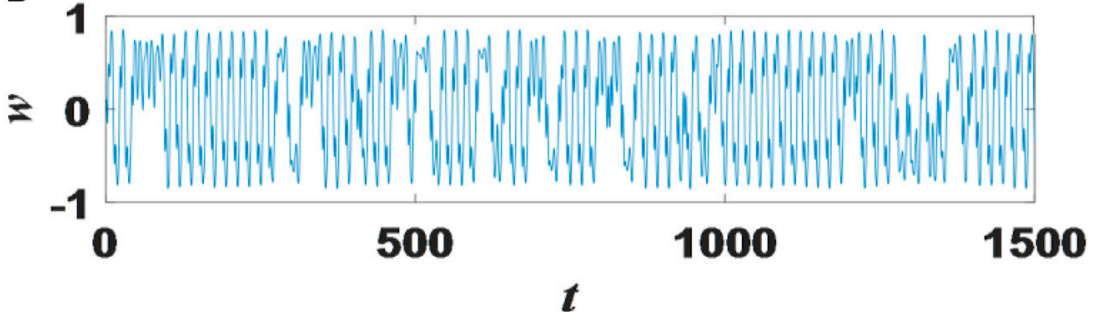

FIGURE 3 | Display the time series diagram of state variables $x$ and $w$. (A) the time series of state variables $x$ (B) the time series of state variables $w$.
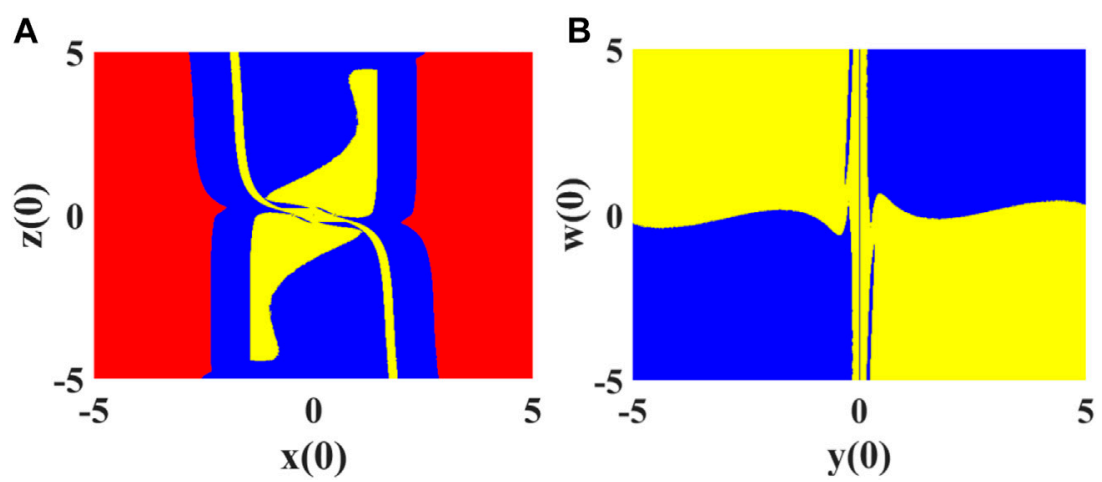

FIGURE 4 | The basin of attraction on the plane: $(\mathbf{A})$ is the basin of attraction on the $x(0)-z(0)$ plane; (B) is the basin of attraction on the $y(0)-w(0)$ plane.

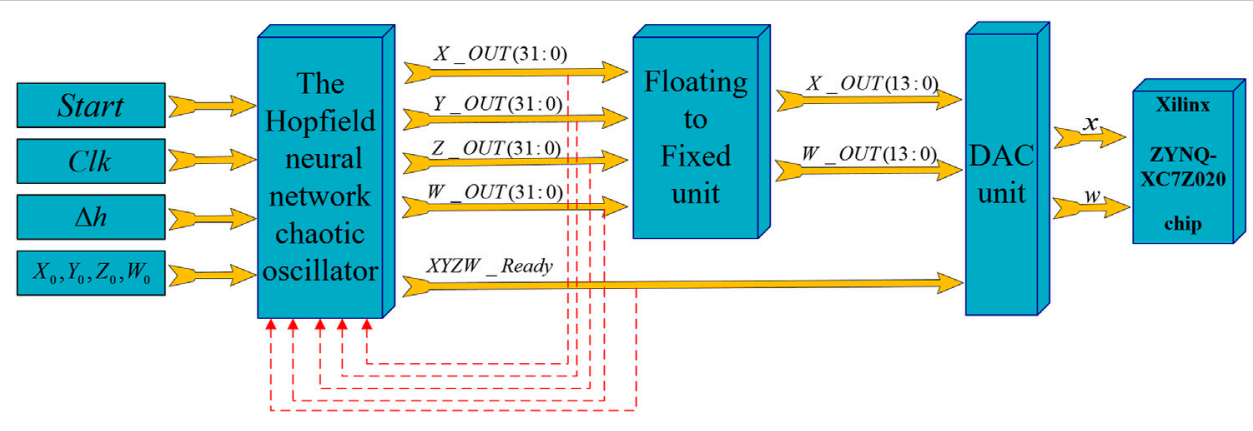

FIGURE 5 | The flow block diagram of the FPGA-based Hopfield neural network system.

Figure 8. In the entropy source unit, the lyapunov exponent of the first dimension of Eq. $\mathbf{1}$ is the largest, indicating that the sensitivity of neuron $x$ is higher, and the change of neuron $x$ will cause the change of other neurons and the magnetic flux passing through neurons.
Therefore, in order to solve the chaotic degradation problem brought by FPGA implementation, the magnetic flux $w$ of neuron $x$ is used as a judgment condition in the feedback controller to selectively interfere with neuron $x$. The specific steps are as follows. 

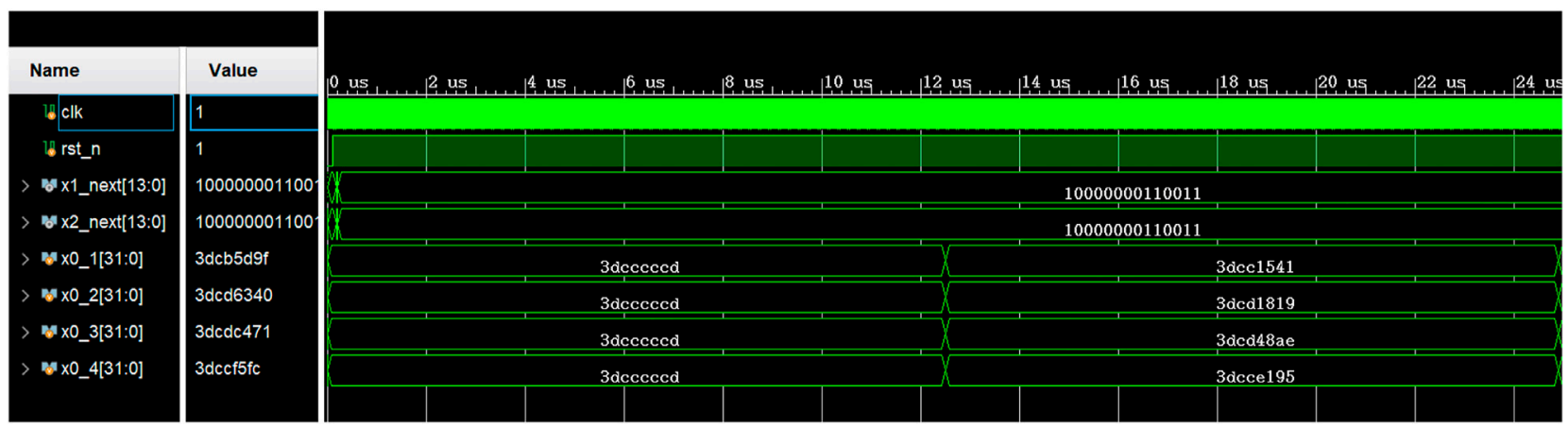

FIGURE 6 | The behavior simulation results given by the vivado platform simulator.
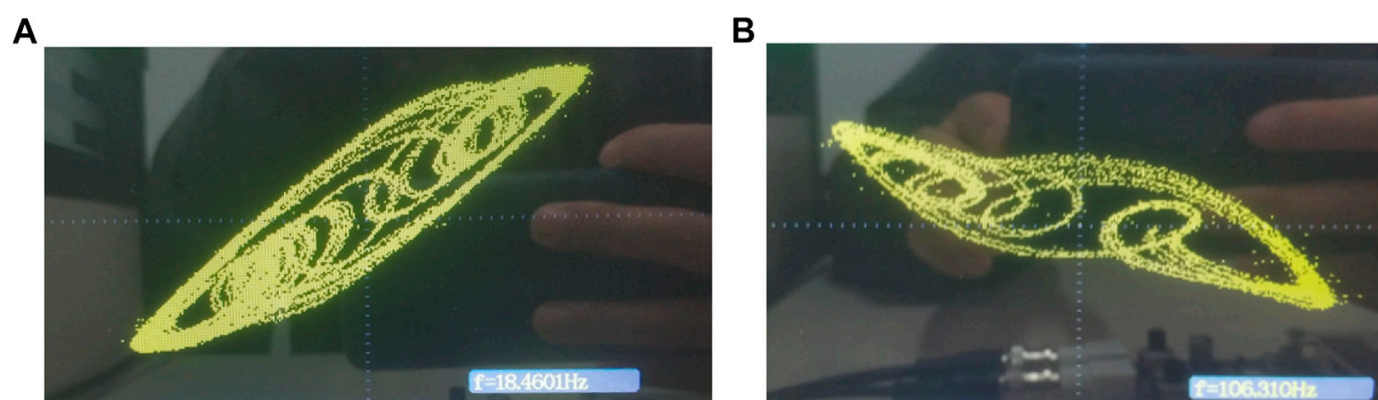

C

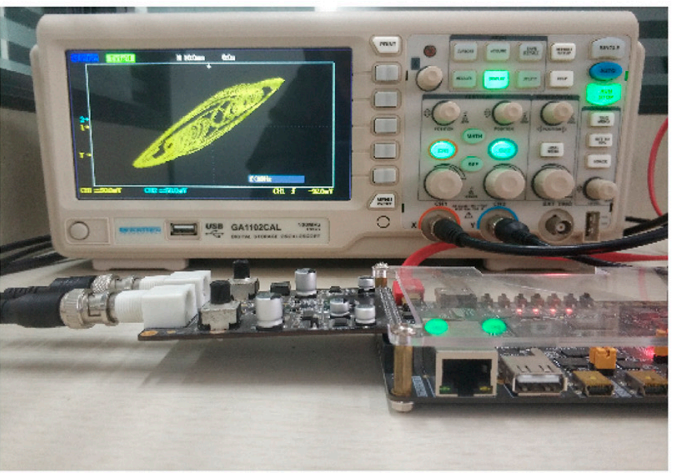

D

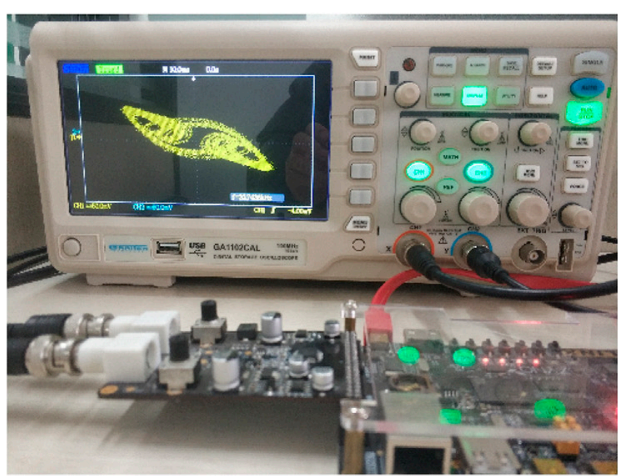

FIGURE 7 | The oscilloscope shows the experimental results of (A) FPGA-based $x-w$ plane phase diagram; (B) FPGA-based $y-w$ plane phase diagram; (C) and (D) indicate the experimental setup of (A) and (B), respectively.

Step 1. Acquire the 32-bit output signal of the magnetic flux W_OUT and the 32-bit output signal of the neuron $X_{-} O U T$. Step 2. Take the 16 th and 17 th bit of $W_{-} O U T$ for XOR operation to get 1 -bit output $w^{\prime}$. If $w^{\prime}$ is equal to 1 , let $X_{1-} O U T=X_{-} O U T+0.0002 ; \quad$ Otherwise, let $X_{1-O U T}=$ $X_{-} O U T-0.0002$.

The output of the feedback controller is given to the sampling quantization unit and used as the input value of the next iteration of the Hopfield neural network oscillator. By using this feedback controller, the period of the random sequence generated by the PRNG can be greatly extended, and the quality of the pseudo-random sequence can be improved.
In the sampling quantization unit, according to ieee 7541985 high-precision 32-bit floating-point standard, in each iteration, bits between 0 and 15 are taken from the four output signals ( $\left.X_{1-} O U T, Y_{1-O U T,} Z_{1_{-}} O U T, W_{1-} O U T\right)$ of the feedback controller unit, and four random sequences $(X, Y, Z, W)$ are obtained by quantization. These four random sequences form a random sequence in order from the first to the fourth dimensions and output the random sequence to the postprocessing unit. The post-processing unit can greatly improve the randomness of the random sequence. In this paper, the initial state of the post-processing unit is shown in Figure 9, which consists of 32 registers and 15 XOR comparators. Among them, the first 16 registers are all 0 , and the last 16 registers 


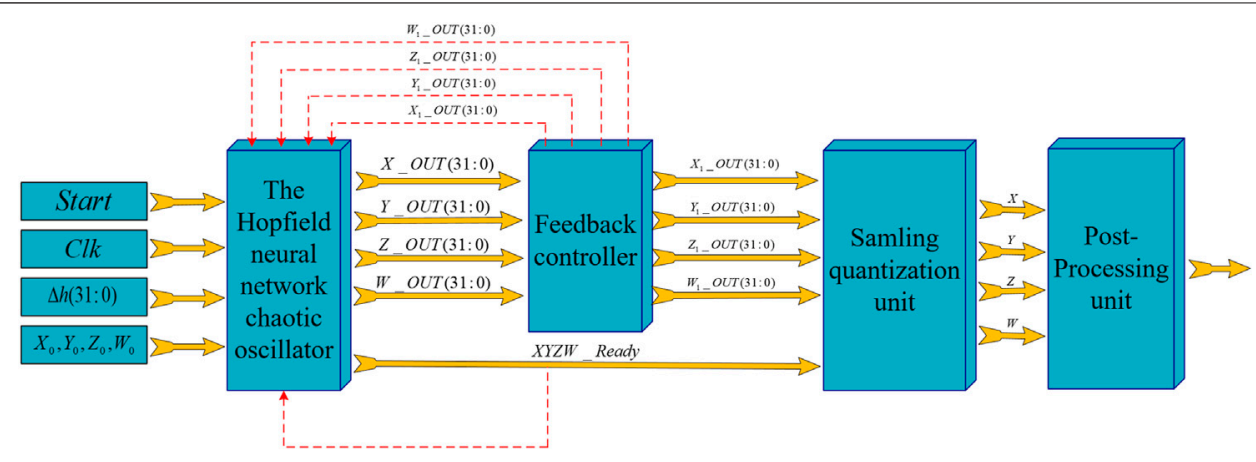

FIGURE 8 | The structure of PRNG.

$\left(k_{0}, k_{1}, k_{2}, \cdots, k_{14}, k_{15}\right)$ are obtained from the random sequence output by the sampling quantization unit in order. The registers are shifted forward one bit at a time, and the XOR operation is completed sequentially. Eventually, every 16 shifts, the last 16-bit of the registers need to be added from the random sequence again. $15 \mathrm{XOR}$ comparators will self-XOR the first 16-bit to generate a 1-bit random number. Until all random sequences are post-processed. Thus, the PRNG generates 64-bit random numbers in each iteration.

\subsection{FPGA Implementation of PRNG}

On the Vivado 2018.3 platform, the simulation results of Hopfield neural network based chaotic oscillator PRNG with feedback controller proposed in this paper on FPGA are shown in Figure 10. Completed by Verilog HDL code. According to the implementation time report, FPGA runs at a clock frequency as high as $109.337 \mathrm{MHz}$, with a minimum running period of $9.146 \mathrm{~ns}$. The data rate of PRNG can reach $16.20 \mathrm{Mbit} / \mathrm{s}$. Table 1 shows the statistics of Xilinx ZYNQ-XC7Z020 chip of PRNG based on FPGA. Finally, the generated bitstream file is output to the oscilloscope, as shown in Figure 11.

\section{SAFETY ANALYSIS}

\subsection{Dynamical Degradation}

In hardware implementation, chaos degradation caused by calculation accuracy will greatly affect the randomness of PRNG. For example, the short period phenomenon may appear in chaotic simulation, which results in periodicity of random sequence, and finally leads to the failure of random sequence test. At present, NIST 800.22 test suite is the most commonly used randomness test standard, which can use 15 test methods to evaluate a large number of random sequences. Therefore, to determine the randomness of the PRNG with a feedback controller based on the Hopfield neural network chaotic oscillator, we tested its generated random sequences using the NIST 800.22 test suite. In this paper, the PRNG discarded the first 50,000 bits of the random sequence and put the resulting 1001 -MIT test random sequences into the NIST 800.22 test suite. The test results of the random sequences generated by the PRNG based on the original Hopfield neural network chaotic oscillator and the random sequence generated by the PRNG based on the Hopfield neural network chaotic oscillator with a feedback controller are shown in Table 2 (a) and (b), respectively. By comparison, three items in the test result (a) show that the $p$ valve is less than 0.01 , and when the $p$ valve is within the range of $[0.01,1]$, it means that the test passed. Therefore, three items in (a) failed NIST 800.22 test suite. Test result (b) shows that all 15 tests have passed, and the random sequence has good randomness. It can be seen that the feedback controller can greatly reduce the impact of chaos degradation on random sequences.

\subsection{Key Space Analysis}

The size of key space is an important index to determine the security of encryption system, and it is very important to choose the right key space. Large key space can improve encryption strength and better resist key analysis. The small key space can not resist exhaustive attack, and the password is easier to be cracked. Usually, when the key space is greater than $2^{128}$, the security of the cipher system can be ensured and the exhaustive attack can be resisted. In this paper, the Hopfield neural network chaotic oscillator and a feedback controller are used to construct PRNG. According to the IEEE 745-1985 floating point standard, the system key consists of the initial conditions $\left(x_{0}, y_{0}, z_{0}, w_{0}\right)$ and the system parameters $(a, b, c, d, e, f, g, h, m, n, \alpha, \beta)$ of the Hopfield neural network chaotic oscillator, totally 512-bits. Therefore, the key space of the system is $2^{512}$, which is much larger than $2^{128}$, and there is enough space to resist the exhaustive attack.

\subsection{Key Sensitivity Analysis}

It is well known that chaotic systems are very sensitive to parameters and initial conditions. Therefore, the proposed PRNG with feedback controller based on Hopfield neural network chaotic oscillator should maintain the same sensitivity. Key sensitivity test is used to analyze the impact of small changes in initial conditions or parameters on the corresponding output. When PRNG has high sensitivity, small changes in the input will lead to huge differences in the corresponding output. In this test, the initial conditions $\left(x_{0}, y_{0}, z_{0}, w_{0}\right)=(0.1,0.1,0.1,0.1)$ and parameters $(\mathrm{a}=3.5, \mathrm{~b}=0.5, \mathrm{c}=2.5, \mathrm{~d}=0.7, \mathrm{e}=3.4, \mathrm{f}=1.6, \mathrm{~g}=0.95$, $\mathrm{h}=2.5, \mathrm{~m}=1.9, \mathrm{n}=1.5, \alpha=0: 24 ; \beta=0: 7)$ of the chaotic system are input into the proposed PRNG to generate a $10^{6}$ bits reference pseudo-random sequence $S_{1}$, and then the initial conditions and parameters are slightly changed as follows. 


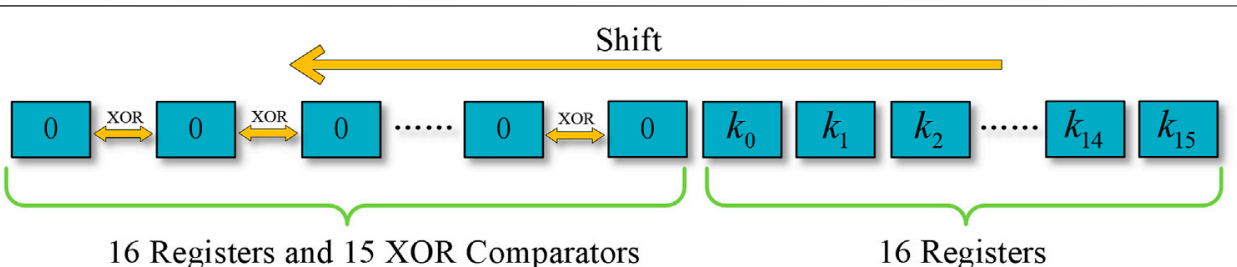

16 Registers and 15 XOR Comparators

16 Registers

FIGURE 9 | Flow chart of the initial state of the post-processing unit.

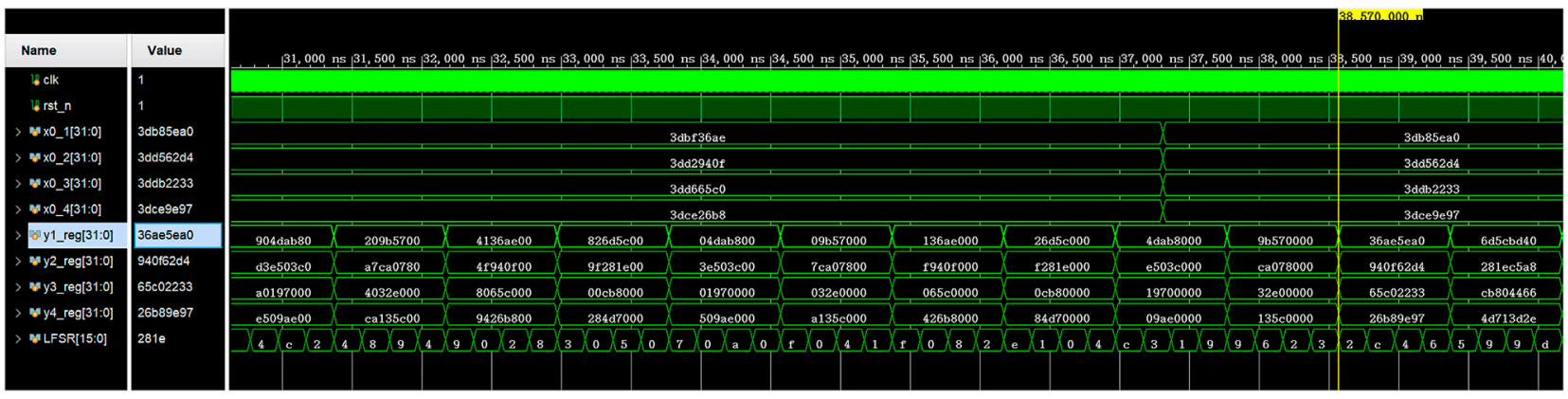

FIGURE 10 | Behavioral simulation results of the Hopfield neural network oscillator-based PRNG with feedback controller.

TABLE 1 | Chip Statistics of PRNG with feedback controller based on Hopfield neural network chaotic oscillator.

\section{Xilinx ZYNQ-XC7Z020 chip statistics}

Used/utilization \%

Number of Slice LUTs

Number of fully used LUT-FF pairs

Number of bonded IOBs

Operating Frequency $(\mathrm{MHz})$

The data rate of PRNG (Mbit/s)

$37,977 / 71.39$

$47,195 / 44.36$

20/16.00

109.337

16.20

1) By modifying the initial condition $x_{0}=0.1$ to $x_{0}^{\prime}=0.1+$ $10^{-8}$ a new test pseudorandom sequence $S_{2}$ with $10^{6}$ bits is obtained.

2) By changing the parameter $a=3.5$ to $a^{\prime}=3.5+10^{-8}$, a new test pseudo-random sequence $S_{3}$ with $10^{6}$ bits is obtained.

The bit change rate has always been considered as an important index to measure the sensitivity of PRNG. The closer the bit change rate is to $50 \%$, the higher the key sensitivity of PRNG. The formula of the corresponding bit change rate is as follows:

$$
P=\frac{\sum_{k=1}^{n}\left|S_{a(k)}-S_{b(k)}\right|}{n} \times 100 \%
$$

where $p$ and $N$ represent the bit change rate and sequence length, $S_{a(k)}$ and $S_{b(k)}$ represent the bit values of the $k$ th bit of the reference random number sequence $S_{a}$ and the test random number sequence $S_{b}$. In this paper, $S_{1}$ is compared with $S_{2}$ and $S_{3}$ respectively, and the bit change rate obtained is shown in Table 3. As can be seen from the table, when the input initial conditions and parameters of the Hopfield neural network chaotic oscillator with feedback controller PRNG increase by only $10^{-8}$, the bit change rate is very close to $50 \%$. This shows that
PRNG is highly sensitive to initial conditions and parameters, and can meet the requirements of security applications. When the initial input condition $x_{0}$ and parameter $a$ change by $10^{-8}$, the time-domain waveform of chaotic oscillator neuron $\mathrm{x}$ of the Hopfield neural network disturbed by the feedback controller is shown in Figure 12. Figure 12A shows the time domain diagram of neuron $x$ when only initial condition $x_{0}$ changes, and Figure 12B shows the time domain diagram of neuron $x$ when only parameter $a$ changes. Thus, the chaotic oscillator of Hopfield neural network disturbed by the feedback controller is very sensitive to the initial conditions and parameters.

\subsection{Correlation Analysis}

Auto-correlation and cross-correlation analysis are important methods to detect the correlation between two random sequences of equal length. Among them, auto-correlation is used to detect the random sequence and its shifted sequence, and cross-correlation is used to detect adjacent test random sequences. Now there are two adjacent random sequences $X=\left\{X_{1}, X_{2}, \cdots, X_{n}\right\}$ and $Y=\left\{Y_{1}, Y_{2}, \cdots, Y_{n}\right\}, X_{i}$ and $Y_{i}$ denote the random numbers in the random sequences $X$ and $Y, i=\{1,2,3, \cdots, n\}$. So the calculation formula of correlation is shown in Eq. 7 .

$$
\begin{aligned}
R_{X Y} & =\frac{\operatorname{Cov}(X, Y)}{S_{X} S_{Y}}=\frac{\frac{\sum_{i=1}^{n}\left(X_{i}-\bar{X}\right)\left(Y_{i}-\bar{Y}\right)}{n-1}}{\sqrt{\frac{\sum_{i=1}^{n}\left(X_{i}-\bar{X}\right)^{2}}{n-1}} \sqrt{\frac{\sum_{i=1}^{n}\left(Y_{i}-\bar{Y}\right)^{2}}{n-1}}} \\
& =\frac{\sum_{i=1}^{n}\left(X_{i}-\bar{X}\right)\left(Y_{i}-\bar{Y}\right)}{\sqrt{\sum_{i=1}^{n}\left(X_{i}-\bar{X}\right)^{2}} \sqrt{\sum_{i=1}^{n}\left(Y_{i}-\bar{Y}\right)^{2}}}
\end{aligned}
$$

where the correlation coefficient $R_{X Y} \in(-1,1)$. The closer the $R_{X Y}$ is to 0 , the more independent the sequence is, and the closer the $R_{X Y}$ is 
A

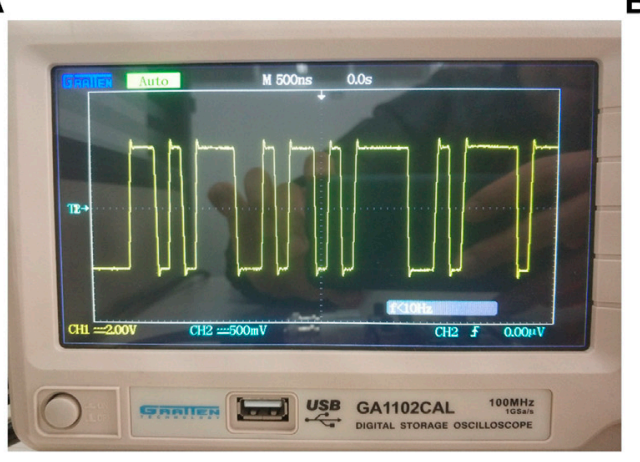

B

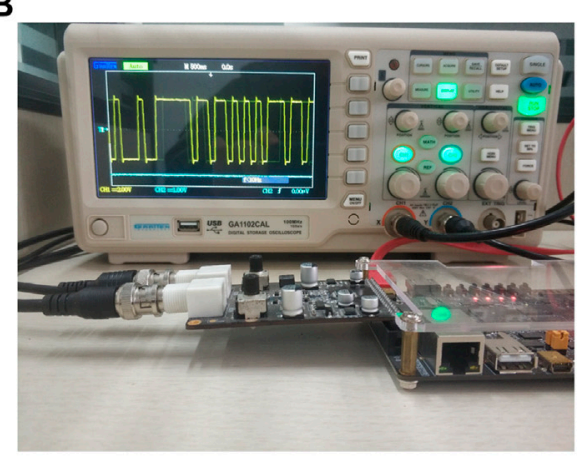

FIGURE 11 | (A) Oscilloscope waveform of PRNG based on FPGA implementation; (B) Experimental equipment.

TABLE 2 | (A) shows the randomness test results of random sequences generated by PRNG based on the original Hopfield neural network chaotic oscillator, and (B) shows the randomness test results of random sequences generated by PRNG with a feedback controller based on the Hopfield neural network chaotic oscillator.

\begin{tabular}{|c|c|c|c|c|c|c|}
\hline Statistical test & (a) Proportion & P-valve & Results & (b) Proportion & P-valve & Results \\
\hline Frequency & 1 & $0.249,284$ & Success & 0.99 & $0.108,791$ & Success \\
\hline BlockFrequency & 0.99 & 0.001030 & Failure & 0.98 & $0.401,199$ & Success \\
\hline CumulativeSums & 0.99 & 0.080519 & Success & 0.99 & $0.759,756$ & Success \\
\hline Runs & 0.99 & $0.779,188$ & Success & 0.99 & $0.494,392$ & Success \\
\hline LongestRun & 1 & 0.037566 & Success & 1 & $0.719,747$ & Success \\
\hline Rank & 0.98 & $0.637,119$ & Success & 1 & $0.334,538$ & Success \\
\hline $\mathrm{FFT}$ & 1 & $0.719,747$ & Success & 1 & $0.991,468$ & Success \\
\hline Non Over lapping Template & 0.95 & $0.983,453$ & Success & 1 & $0.998,821$ & Success \\
\hline Over lapping Template & 0.97 & $0.275,709$ & Success & 0.98 & $0.419,021$ & Success \\
\hline Universal & 1 & $0.983,453$ & Success & 0.98 & 0.032923 & Success \\
\hline ApproximateEntropy & 0.92 & 0.000000 & Failure & 0.97 & $0.129,620$ & Success \\
\hline RandomExcursions & 1 & $0.982,743$ & Success & 1 & $0.999,438$ & Success \\
\hline RandomExcursionsVariant & 1 & 0.012650 & Success & 1 & $0.568,055$ & Success \\
\hline Serial & 0.94 & 0.000000 & Failure & 0.99 & $0.108,791$ & Success \\
\hline LinearComplexity & 0.98 & $0.595,549$ & Success & 0.99 & $0.897,763$ & Success \\
\hline
\end{tabular}

to 1 or -1 , the stronger the positive or negative correlation between the sequences. $\operatorname{Cov}(X, Y)$ is the covariance of the sequence, and $S_{X}$ and $S_{Y}$ are the standard deviations of the sequence. $n$ denotes the length of the sequence and $\bar{X}=\sum_{i=1}^{n} \frac{X_{i}}{n}, \bar{Y}=\sum_{i=1}^{n} \frac{Y_{i}}{n}$. The calculated correlation coefficient $R_{X Y}=2.07 \times 10^{-4}$, so it can be considered that there is no correlation between the two test random sequences. Autocorrelation detection and cross-correlation detection are shown in Figure 13. Figure 13A shows the auto-correlation between test random sequences and its shifted sequences generated by 15 keys, and Figure 13B shows the cross-correlation between test random numbers generated by 15 adjacent keys. It can be seen from the figure that there is no correlation between pseudo-random sequences generated by PRNG proposed in this paper.

\section{DESIGN AND IMPLEMENTATION OF IMAGE ENCRYPTION AND DECRYPTION SYSTEM BASED ON PRNG}

In recent years, image and video encryption based on chaotic system has been widely studied and applied [51-56]. As the main application of chaotic system, PRNG has been paid more
TABLE 3 | The bit change rate of random number sequence $S_{2}$ and. $S_{3}$

\begin{tabular}{lccc} 
& $\Delta \mathbf{x}=\mathbf{x}_{\mathbf{0}}^{\prime}-\mathbf{x}_{\mathbf{0}}$ & $\boldsymbol{\Delta} \mathbf{a}=\mathbf{a}^{\prime}-\mathbf{a}$ & $\boldsymbol{P ( \% )}$ \\
\hline$S_{2}$ & $10^{-8}$ & 0 & $49.96 \%$ \\
$S_{3}$ & 0 & $10^{-8}$ & $50.00 \%$
\end{tabular}

and more attention in the field of image encryption [57-59]. At the same time, FPGA also provides strong support for the engineering application of chaotic system [60]. Therefore, as the basis of different engineering applications based on PRNG, FPGA has been paid more and more attention. It is understood that the PRNG with feedback controller based on Hopfield neural network chaotic oscillator proposed in this paper has a complex mathematical model and requires a large amount of chip resources in FPGA implementation. Currently, there is no image encryption system based on this PRNG implemented by FPGA.

\subsection{System Simulation and Security Analysis 5.1.1 System Simulation}

In this section, we propose an image encryption system based on the pseudo-random sequences generated by PRNG, and complete the simulation and security analysis on matlab platform. The 

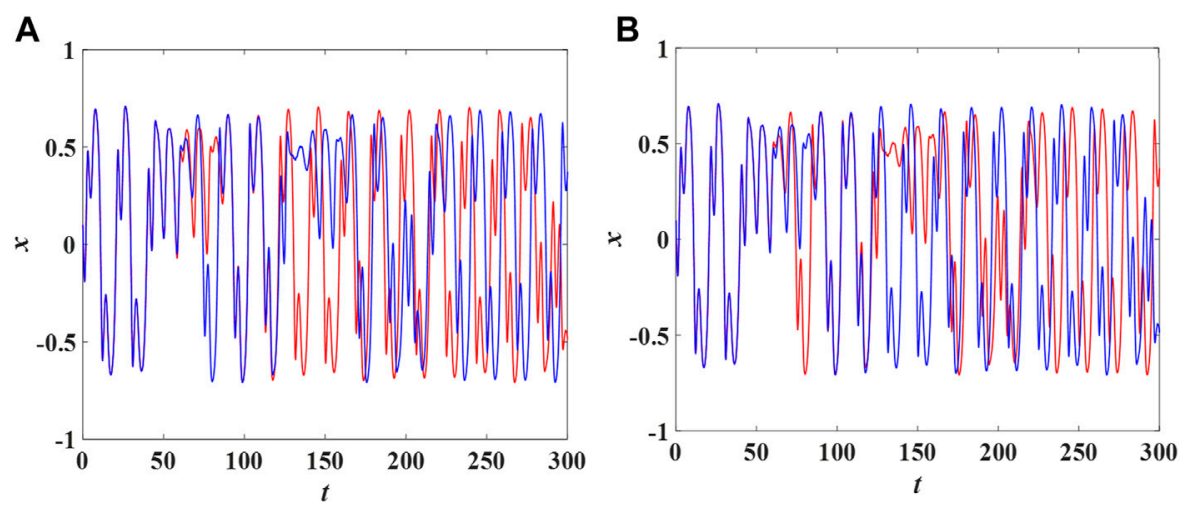

FIGURE 12 | (A) is the time domain diagram of neuron $x$ when only initial condition $x_{0}$ changes; (B) is the time domain diagram of neuron $x$ when only parameter a changes.
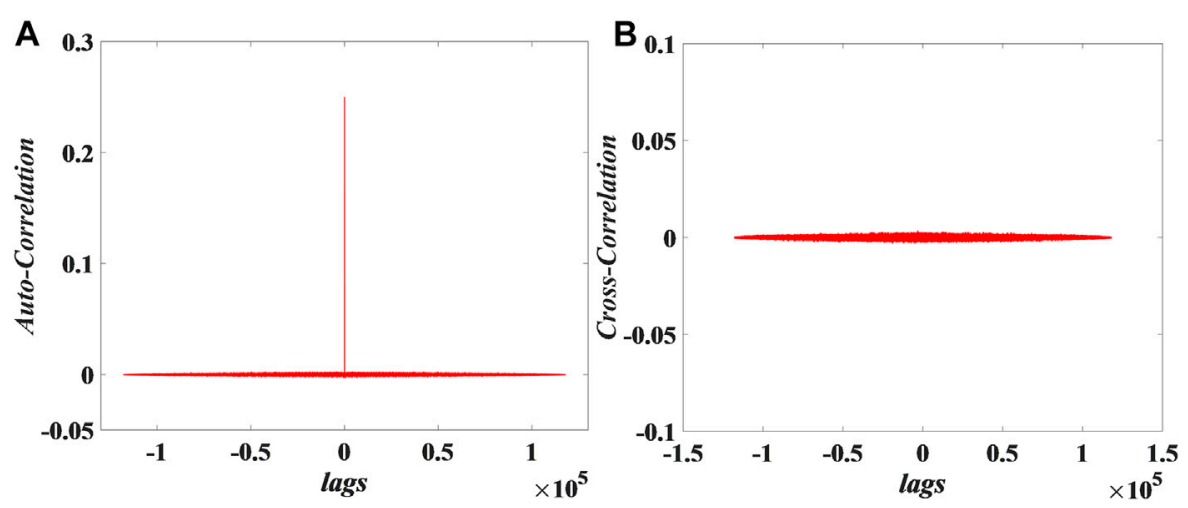

FIGURE 13 | Correlation analysis of 15 sequences for PRNG: (A) Auto-correlation, (B) Cross-correlation.

encryption and decryption scheme of the image encryption system is as follows:

Step 1: A $256 \times 25624$-bit depth true color image "Baboon" is selected as the original plaintext image $p$, and the $256 \times 25624$ bit pixels are divided into three $256 \times 2568$-bit depth $R, G$ and $B$ pixel channels.

Step 2: The generation of the key sequence used for encryption is consistent with our previous work. In each iteration, a 64-bit random sequence will be generated and the last 8 bits of the random sequence will be kept and added to the key sequence. Finally, three $256 \times 2568$-bit key sequences $S_{1}, S_{2}$ and $S_{3}$ are generated.

Step 3: We get the $R, G$ and $B$ pixel channels from step 1 and the key sequence $S_{1}, S_{2}$ and $S_{3}$ from step 2 for XOR processing, that is, the pixel channels of the encrypted image are $R_{1}=R \oplus S_{1}, G_{1}=G \oplus S_{2}, B_{1}=B \oplus S_{3}$. The reverse process of encryption is the decryption process.

The experimental results of encrypting the original plaintext image $p$ using key sequences $S_{1}, S_{2}$ and $S_{3}$ are shown in Figure 14. Figures 14A,B represent the original image and the encrypted image, respectively. When the encrypted image has the correct key sequence, the original image can be obtained, as shown in Figure 14C.

\subsubsection{Security Analysis}

In this section, we will conduct a security analysis to evaluate the proposed image encryption and decryption system. Security analysis includes histogram analysis, correlation analysis, differential key attack analysis, and entropy analysis. The master key consists of parameters $a=3.5, b=0.5, c=2.5, d=0.7, e=$ $3.4, f=1.6, \quad g=0.95, h=2.5, m=1.9, n=1.5, \alpha=0.24, \beta=0.7$ and initial conditions $(0.1,0.1,0.1,0.1)$. Set the PRNG step $\Delta h=$ 0.001 and iterate $(3 \times 256 \times 256+500)$ times. Finally, the results discard the results of the first 500 iterations.

1) Histogram analysis: The intensity of the distribution of the image pixel values can be known from the histogram. In general, the ideal histogram distribution should be uniform. Therefore, a high-security image encryption and decryption system can make the encrypted image have the ideal histogram distribution. The histograms of the original 

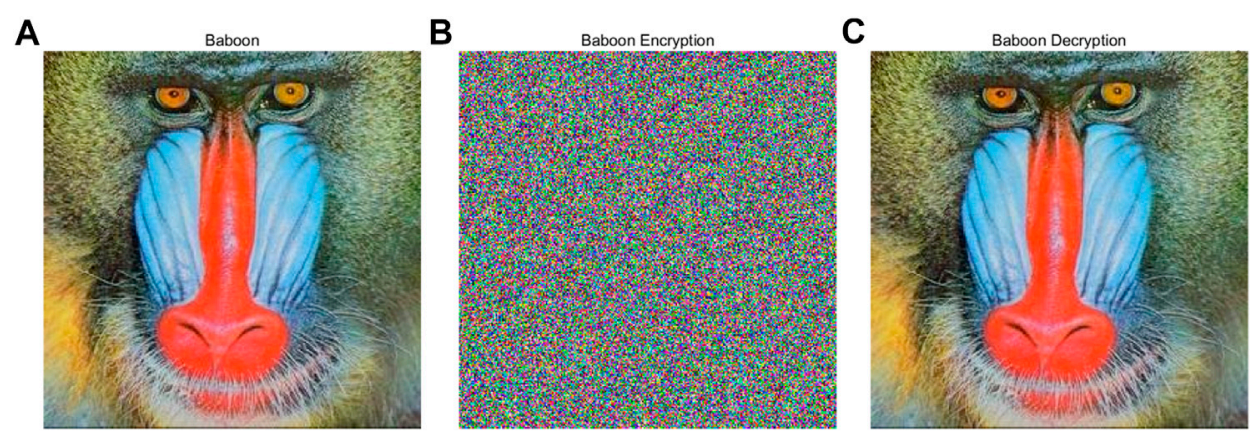

FIGURE 14 | (A) original image; (B) encrypted image; (C) decrypted image.

plaintext image and its $R, G$ and $B$ pixel channels are shown in Figures 15A-D. The histograms of the encrypted image and its $R_{1}, G_{1}$ and $B_{1}$ pixel channels are shown in Figures 15E-H, respectively. The results show that the histogram of the encrypted image is the ideal histogram distribution, which prevents the attacker from obtaining information from the histogram.

2) Correlation analysis: The quality of the image encryption and decryption system is related to the correlation between adjacent pixels of the encrypted image. The adjacent pixels of the original plaintext image have a high correlation. Therefore, a good image encryption and decryption system can effectively reduce the correlation coefficient between adjacent pixels. In this experiment, we randomly selected 10,000 pairs of adjacent pixels in the horizontal, vertical and diagonal directions to calculate the correlation coefficients of the original plaintext image and the encrypted image. The correlation coefficients can be calculated by Eq. 7, and the results are shown in Table 4 . The results show that the system has the ability to resist statistical attacks.

3) Differential key attack analysis: Differential key attack analysis is an important method to evaluate the resistance of image encryption and decryption systems to attacks. Among them, number of pixel change rate (NPCR) and unified average changing intensity (UACI) are used as metrics for the analysis. Suppose we generate two key sequences $S_{1}$ and $S_{2}$ respectively using the correct key and the wrong key with the parameter increased by $10^{-8}$. The original plaintext image is encrypted by $S_{1}$ and $S_{2}$ to obtain two encrypted images $T_{1}$ and $T_{2}$, and the pixel values of $T_{1}$ and $T_{2}$ are represented by $T_{1}(i, j)$ and $T_{2}(i, j)$. the mathematical formulas of NPCR and UACI are given by Eqs. 8-10

$$
\begin{gathered}
N P C R=\sum_{i=1}^{N} \sum_{j=1}^{M} \frac{D(i, j)}{N \times M} \times 100 \% \\
D(i, j)=\left\{\begin{array}{l}
1, T_{1}(i, j) \neq T_{2}(i, j) \\
0, T_{1}(i, j)=T_{2}(i, j)
\end{array}\right. \\
U A C I=\sum_{i=1}^{N} \sum_{j=1}^{M} \frac{\left|T_{1}(i, j)-T_{2}(i, j)\right|}{256 \times N \times M} \times 100 \%
\end{gathered}
$$

Where, $N$ and $M$ denote the number of pixels in the width and length of the encrypted image. The calculation results are shown in Table 5, and the results are ideal.

4) Entropy analysis: Judging the security of an image encryption and decryption system requires the help of information entropy. When the information entropy of an encrypted image is close to 8 , we say that it achieves the ideal information entropy and indicates that the encryption and decryption system has good security. The formula for calculating information entropy is as follows.

$$
H(t)=\sum_{i=0}^{2^{N}-1} P\left(t_{i}\right) \log _{2} \frac{1}{P\left(t_{i}\right)},
$$

The results are shown in Table 6, and the entropy value of the encrypted image is close to 8 , reaching the ideal information entropy.

\subsection{FPGA-Based Image Encryption and Decryption System}

In this section, we design and implement a PRNG with a feedback controller image encryption system based on the Hopfield neural network chaotic oscillator on FPGA. All experiments also adopt 32-bit IEEE 754-1985 floating-point standard, design and simulation on Vivado 2018.3 platform using Verilog HDL hardware language and developed IP-core generator, and finally, completed on Xilinx Zynq-XC7Z020 chip. The key sequence generation and image encryption and decryption processes are consistent with the simulation process. Figure 16. is the flow chart of implementing PRNG based image encryption system on FPGA. As shown in Figure 16, the image encryption system on FPGA consists of four parts: chip data RAM, the key sequence, data encryption module and VGA display controller. Where the image data and random sequence accessed in the chip are provided by the software Image2Lcd and the PRNG proposed in this paper, respectively. The encrypted image data will be stored in the chip and processed with the random sequence again. The image decryption system consists of 

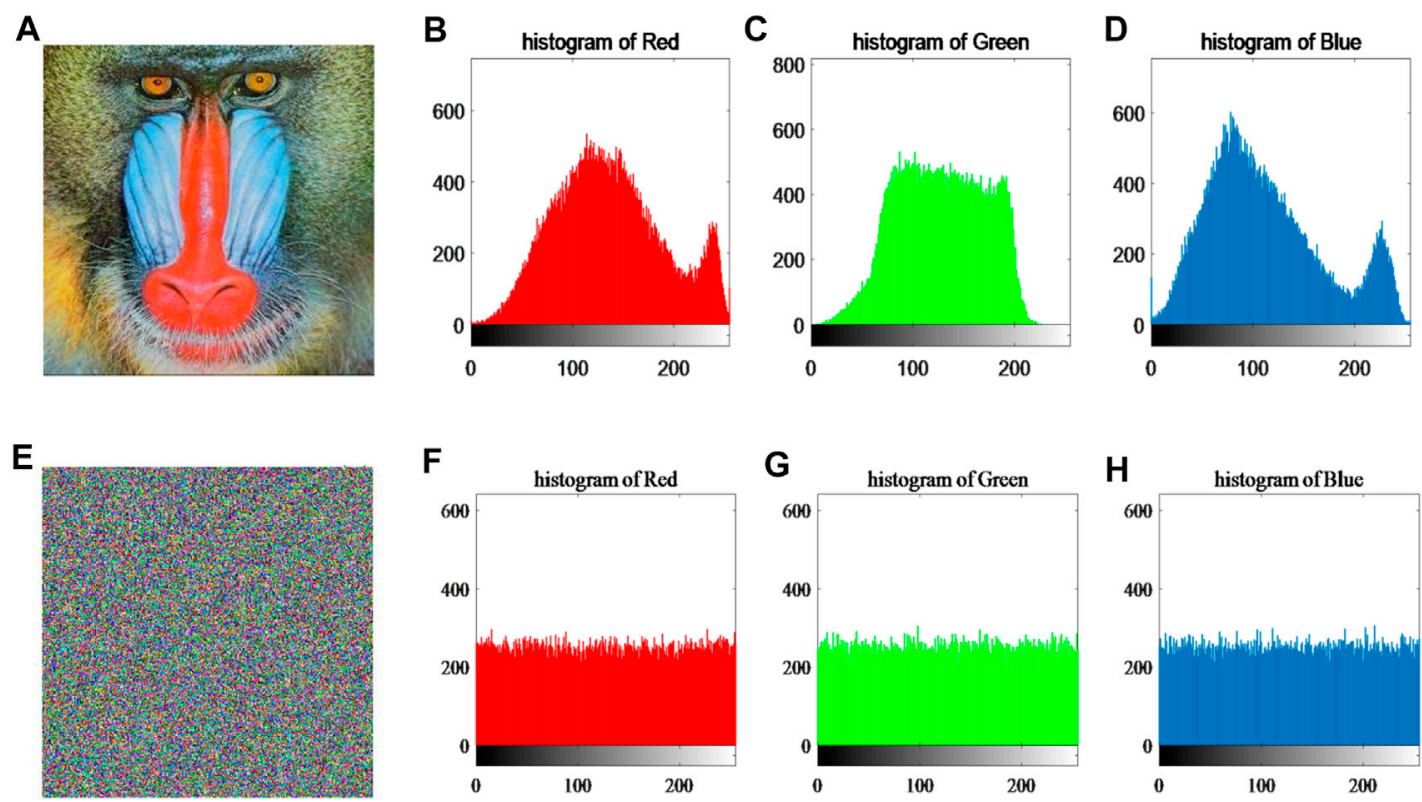

FIGURE 15 | The histogram simulation results of the original image and encrypted image. (A) original image; (B), (C) and (D) are histograms of $R, G$ and $B$ pixel channels of the original image, respectively; $(\mathbf{E})$ encrypted image; $\mathbf{( F ) , ~ ( G ) ~ a n d ~} \mathbf{( H )}$ are histograms of $R, G$ and $B$ pixel channels of the encrypted image, respectively.

TABLE 4 | Correlation coefficient analysis results.

\begin{tabular}{lccc}
\hline Image & Horizontal & Vertical & Diagonal \\
\hline Original image & 0.8650 & 0.8975 & 0.8372 \\
Encrypted image & -0.0076 & -0.0019 & 0.0132
\end{tabular}

TABLE 5 | NPCR and UACl detection results of two encrypted images.

\begin{tabular}{ll} 
Image & Baboon \\
\hline NPCR (\%) & 99.5575 \\
UACI (\%) & 33.3993
\end{tabular}

three modules: the key sequence, the data decryption module and the VGA display controller. In this experiment, the Image2Lcd software is used to divide the $256 \times 25624$-bit depth true color image "Baboon" into three $256 \times 256$ 8-bit data and store them in the chip data RAM. In the data encryption module, the image data is XORed with the random sequence, and the calculated data is transmitted to the display through the VGA display controller to complete the image encryption. Figure 17. shows the experimental results based on FPGA. As shown in Figure 17A, the encrypted image is obtained by processing the data of the original image and the key sequence. Figure 17B shows that when the encrypted image data is processed with the correct key sequence, the original image before encryption can be obtained. The experimental results verify the value of the proposed PRNG in engineering application.
TABLE 6 | The results of information entropy about $R, B, G$ and total pixel channel of original plaintext image and encrypted image.

\begin{tabular}{lcc} 
Pixel channel & Original image & Encrypted image \\
\hline R & 7.6959 & 7.9975 \\
G & 7.3907 & 7.9974 \\
$B$ & 7.6985 & 7.9972 \\
Total & 7.7050 & 7.9992
\end{tabular}

\section{CONCLUSION}

In this paper, a PRNG with a feedback controller based on the improved Hopfield chaotic neural network oscillator is proposed and well implemented on FPGA. Among them, the magnetic flux of neurons is taken as the judgment condition, and the feedback controller is used to add the corresponding interference factor to the neurons with the highest Lyapunov exponent, so as to reduce the influence of chaos degradation on the generated random numbers and improve the randomness of the random sequence. The post-processing unit consists of 32 registers and 15 XOR comparators. From the chip statistics, it can be seen that the PRNG can be implemented on FPGA and the output data rate can be up to $16.2 \mathrm{Mbit} / \mathrm{s}$. The performance of the PRNG was tested. The security analysis and FPGA implementation of the image encryption and decryption system based on PRNG show that PRNG has good randomness and engineering application value. Existing feedback controllers and post-processing algorithms will be improved in the future to further improve the randomness of the PRNG and reduce the impact of chaotic degradation. 


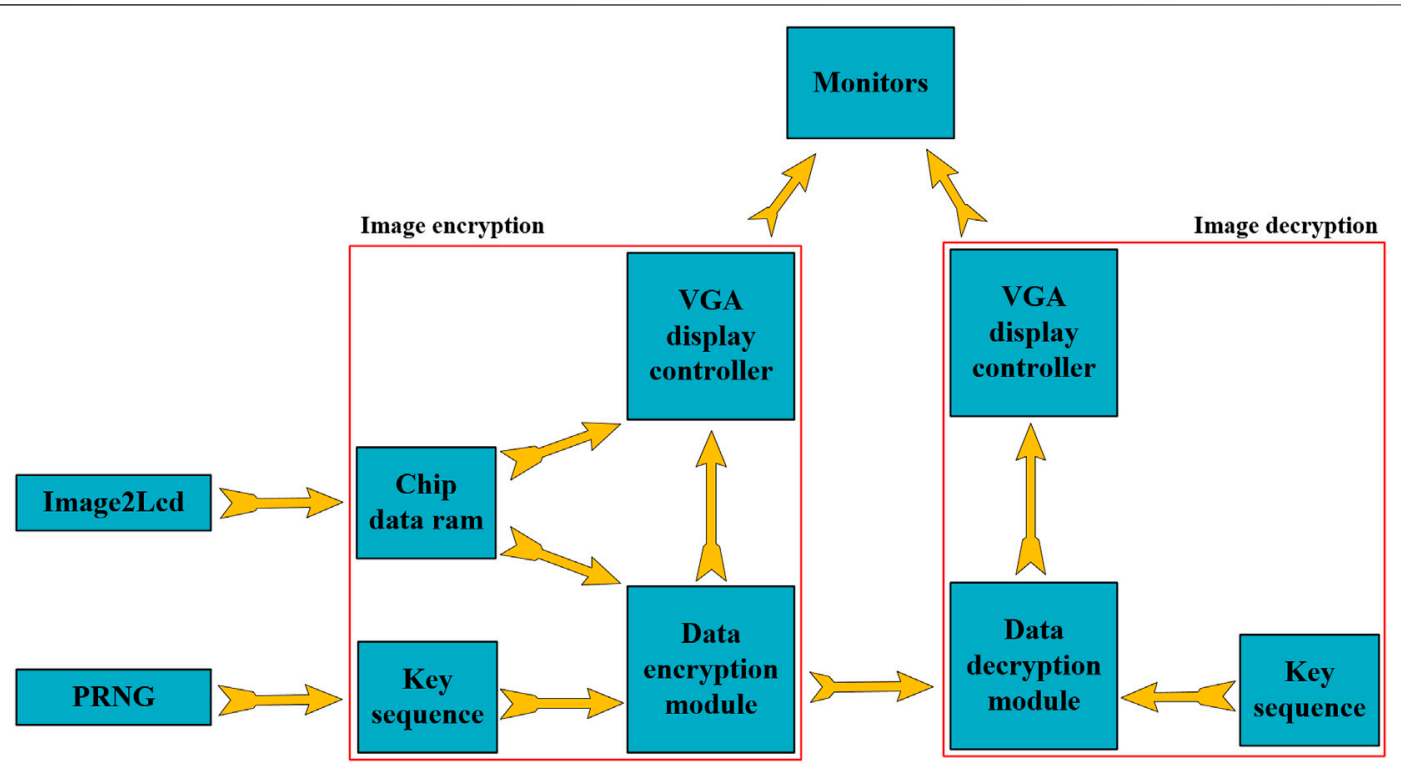

FIGURE 16 | The structure of image encryption and decryption system based on FPGA.
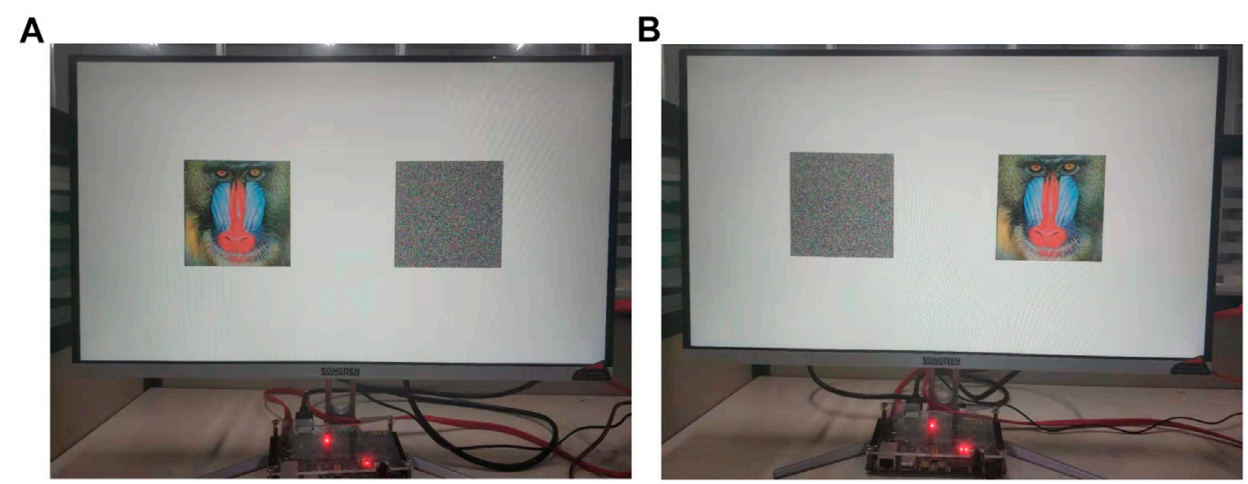

FIGURE 17 | The results of image encryption and decryption based on PRNG with feedback controller. (A) the original and encrypted image on the FPGA. (B) the encrypted and decrypted image on the FPGA.

\section{DATA AVAILABILITY STATEMENT}

The raw data supporting the conclusion of this article will be made available by the authors, without undue reservation.

\section{AUTHOR CONTRIBUTIONS}

All authors listed have made substantial, direct, and intellectual contribution to the work and approved it for publication.

\section{FUNDING}

This work was supported by the National Natural Science Foundation of China under Grants No. 61504013 and
61702052, and by the Natural Science Foundation of Hunan Province under Grants No. 2019JJ50648, 2020JJ4622, and 2020JJ4221, and by Guangxi Key Laboratory of Cryptography and Information Security under Grant No. GCIS201919, and by the Postgraduate Training Innovation Base Construction Project of Hunan Province under Grant No. 2020-172-48, and the Postgraduate Scientic Research Innovation Project of Hunan Province under Grant No. CX20200884, and by the Scientific Research Fund of Hunan Provincial Education Department under grant no. 18A137, and by the young teacher development program project of Changsha university of science and technology under grant 2019QJCZ013, and by the special funds for the construction of innovative provinces in Hunan Province under grant 2020JK4046. 


\section{REFERENCES}

1. Zhong S. Heterogeneous Memristive Models Design and its Application in Information Security. Computer Mater Continua (2019) 60(2):465-79. doi:10. 32604/cmc.2019.05853

2. Zuo J, Lu Y, Gao H, Cao R, Guo Z, and Feng J. Comprehensive Information Security Evaluation Model Based on Multi-Level Decomposition Feedback for Iot. Comp Mater Continua (2020) 65(1):683-704. doi:10.32604/cmc.2020. 010793

3. Fang Z, Cai J, and Tian L. Security of Chip Bank Card in Remote Payment Based on Risk Feature. Comp Syst Sci Eng (2020) 35(4):299-305. doi:10.32604/ csse.2020.35.299

4. Du Z. Personal Data Security and Supervision in the Age of Large Data. Intell Automation Soft Comput (2019) 25(4):847-53.

5. Centonze P. Security and Privacy Frameworks for Access Control Big Data Systems. Comp Mater Continua (2019) 59(2):361-74. doi:10.32604/cmc.2019. 06223

6. Gu K, Wu N, Yin B, and Jia WSecure Data Query Framework for Cloud and Fog Computing. IEEE Trans Netw Serv Manag (2019) 17(1):332-45.

7. Han W, Tian Z, Huang Z, Zhong L, and Jia Y. System Architecture and Key Technologies of Network Security Situation Awareness System Yhsas. Comp Mater Continua (2019) 59(1):167-80. doi:10.32604/cmc.2019. 05192

8. You I, Choi C, Sharma V, Woungang V, Bhargava I, and Bharat K Guest Editorial: Advances in Security and Privacy Technologies for Forthcoming Smart Systems, Services, Computing, and Networks. Intell Automation Soft Comput (2019) 25(1):117-9.

9. Kelec A, and Djuric Z. A Proposal for Addressing Security Issues Related to Dynamic Code Loading on Android Platform. Comp Syst Sci Eng (2020) 35(4): 271-82. doi:10.32604/csse.2020.35.271

10. Zhang Q, Liang Z, and Cai Z. Developing a New Security Framework for Bluetooth Low Energy Devices. Comput Mater Continua (2019) 59(2):457-71. doi:10.32604/cmc.2019.03758

11. Yu F, Qian S, Chen X, Huang S, Cai Y, Jin S, et al. Chaos-based Engineering Applications with a 6d Memristive Multistable Hyperchaotic System and a 2d Sf-Simm Hyperchaotic Map. Complexity (2021) 2021:6683284. doi:10.1155/ 2021/6683284

12. Murillo-Escobar MA, Cruz-Hernández C, Cardoza-Avendaño L, and MéndezRamírez R. A Novel Pseudorandom Number Generator Based on Pseudorandomly Enhanced Logistic Map. Nonlinear Dyn (2017) 87(1): 407-25. doi:10.1007/s11071-016-3051-3

13. Deng Q, Wang C, and Yang L. Four-wing Hidden Attractors with One Stable Equilibrium point. Int J Bifurcation Chaos (2020) 30(06):2050086. doi:10.1142/ s0218127420500868

14. Yu F, Shen H, Zhang Z, Huang Y, Cai S, and Du S. A New Multi-Scroll Chua's Circuit with Composite Hyperbolic tangent-cubic Nonlinearity: Complex Dynamics, Hardware Implementation and Image Encryption Application. Integration (2021), 2021 in press.

15. Yang F, Mou J, Ma C, and Cao Y. Dynamic Analysis of an Improper Fractional-Order Laser Chaotic System and its Image Encryption Application. Opt lasers Eng (2020) 129:106031. doi:10.1016/j.optlaseng. 2020.106031

16. Yu F, Shen H, Liu L, Zinan Z, Yuanyuan H, Binyong H, et al. "Ccii and Fpga Realization: A Multistable Modified Fourth-Order Autonomous Chua's Chaotic System with Coexisting Multiple Attractors. Complexity (2020) 2020:5212601. doi:10.1155/2020/5212601

17. Wan Q, Zhou Z, Ji W, Wang C, Yu F, et al.Dynamic Analysis and Circuit Realization of a Novel No-Equilibrium 5d Memristive Hyperchaotic System with Hidden Extreme Multistability. Complexity (2020) 2020:7106841. doi:10. $1155 / 2020 / 7106861$

18. Wen $\mathrm{Z}$, Li Z, and $\mathrm{Li} \mathrm{X}$. Bursting Dynamics in Parametrically Driven Memristive Jerk System. Chin J Phys (2020) 66:327-34. doi:10.1016/j.cjph. 2020.04.009

19. Yao W, Wang C, Sun Y, and Yao W (2020). Robust Multimode Function Synchronization of Memristive Neural Networks with Parameter Perturbations and Time-Varying Delays. IEEE Trans Syst Man, Cybernetics: Syst. doi:10.1109/TSMC.2020.2997930
20. Zhou C, Wang C, Sun Y, et al. Weighted Sum Synchronization of Memristive Coupled Neural Networks. Neurocomputing (2020) 403:225-32. doi:10.1016/j. neucom.2020.04.087

21. Yu F, Qian S, Chen X, Huang Y, Liu L, Shi C, et al. A New 4d Four-wing Memristive Hyperchaotic System: Dynamical Analysis, Electronic Circuit Design, Shape Synchronization and Secure Communication. Int J Bifurcation Chaos (2020) 30(10):2050147. doi:10.1142/s0218127420501473

22. Li Y, Li Z, Ma M, and Wang M. Generation of Grid Multi-wing Chaotic Attractors and its Application in Video Secure Communication System. Multimedia Tools Appl (2020) 79:29161-77. doi:10.1007/s11042020-09448-7

23. Xiu C, Zhou R, Zhao S, and Xu G. Memristive Hyperchaos Secure Communication Based on Sliding Mode Control. Nonlinear Dyn (2021) 104:789-805. doi:10.1007/s11071-021-06302-9

24. Lin H, and Wang C. Influences of Electromagnetic Radiation Distribution on Chaotic Dynamics of a Neural Network. Appl Math Comput (2020) 369: 124840. doi:10.1016/j.amc.2019.124840

25. Yao W, Wang C, Cao J, Sun Y, and Zhou C. Hybrid Multisynchronization of Coupled Multistable Memristive Neural Networks with Time Delays. Neurocomputing (2019) 363:281-94. doi:10.1016/j.neucom.2019.07.014

26. Yu F, Liu L, Xiao L, Li K, and Cai S. A Robust and Fixed-Time Zeroing Neural Dynamics for Computing Time-Variant Nonlinear Equation Using a Novel Nonlinear Activation Function. Neurocomputing (2019) 350:108-16. doi:10. 1016/j.neucom.2019.03.053

27. Yao W, Wang C, Sun Y, Zhou C, and Lin H. Synchronization of Inertial Memristive Neural Networks with Time-Varying Delays via Static or Dynamic Event-Triggered Control. Neurocomputing (2020) 404:367-80. doi:10.1016/j. neucom.2020.04.099

28. Xu Q, Tan X, Zhu D, Bao H, Hu Y, and Bao B. Bifurcations to Bursting and Spiking in the Chay Neuron and Their Validation in a Digital Circuit. Chaos, Solitons \& Fractals (2020) 141:110353. doi:10.1016/j.chaos.2020.110353

29. Lin H, Wang C, and Tan Y. Hidden Extreme Multistability with Hyperchaos and Transient Chaos in a Hopfield Neural Network Affected by Electromagnetic Radiation. Nonlinear Dyn (2020) 99(3):2369-86. doi:10. 1007/s11071-019-05408-5

30. Li Z, Zhou H, Wang M, and Ma M Coexisting Firing Patterns and Phase Synchronization in Locally Active Memristor Coupled Neurons with $\mathrm{Hr}$ and Fn Models. Nonlinear Dyn (2021) 104, 1455-1473. doi:10.1007/s11071-02106315-4

31. Wang F, Zhang L, Zhou S, and Huang Y. Neural Network-Based Finite-Time Control of Quantized Stochastic Nonlinear Systems. Neurocomputing (2019) 362:195-202. doi:10.1016/j.neucom.2019.06.060

32. Long $\mathrm{M}$, and Zeng $\mathrm{Y}$. Detecting Iris Liveness with Batch Normalized Convolutional Neural Network. Comp Mater Continua (2019) 58(2): 493-504. doi:10.32604/cmc.2019.04378

33. Yu F, Li L, He B, Liu L, Qian S, Shen H, et al. Pseudorandom Number Generator Based on a $5 \mathrm{~d}$ Hyperchaotic Four-wing Memristive System and its Fpga Implementation. Eur Phys Journal-Special Top (2021). doi:10.1140/epjs/ s11734-021-00132-x

34. Dong L, and Yao G. Method for Generating Pseudo Random Numbers Based on Cellular Neural Network. J Commun (2016) 37:85-91.

35. Yu F, Li L, He B, Liu L, Qian S, Huang Y, et al. Design and Fpga Implementation of a Pseudorandom Number Generator Based on a Four-wing Memristive Hyperchaotic System and Bernoulli Map. IEEE Access (2019) 7:181 884-181. doi:10.1109/access.2019.2956573

36. Tuna M. A Novel Secure Chaos-Based Pseudo Random Number Generator Based on Ann-Based Chaotic and Ring Oscillator: Design and its Fpga Implementation. Analog Integr Circ Sig Process (2020) 105(2):167-81. doi:10.1007/s10470-020-01703-z

37. Kawashima I, Morie T, and Tamukoh H. Fpga Implementation of HardwareOriented Chaotic Boltzmann Machines. IEEE Access (2020) 8:204360-204.

38. Fraga D, Gerardo L, Esteban T, and Cuauhtemoc M. Hardware Implementation of Pseudo-random Number Generators Based on Chaotic Maps. Nonlinear Dyn (2017) 90(3):1661-70.

39. Chen X, Qian S, Yu F, Zhang Z, Zinan S, Shen H, et al. Pseudorandom Number Generator Based on Three Kinds of Four-wing Memristive Hyperchaotic System and its Application in Image Encryption. Complexity (2020) 2020:8274685. doi:10.1155/2020/8274685 
40. Zhao Y, Gao C, Liu J, and Dong S. A Self-Perturbed Pseudo-random Sequence Generator Based on Hyperchaos. Chaos, Solitons \& Fractals: X (2019) 4: 100023. doi:10.1016/j.csfx.2020.100023

41. Singh JP, Pham VT, Hayat T, Jafari S, Alsaadi FE, and Roy BK. A New FourDimensional Hyperjerk System with Stable Equilibrium point, Circuit Implementation, and its Synchronization by Using an Adaptive Integrator Backstepping Control. Chin Phys. B (2018) 27(10):100501. doi:10.1088/16741056/27/10/100501

42. Liu Y, and Tong X. Hyperchaotic System-based Pseudorandom Number Generator. IET Inf Security (2016) 10(6):433-41. doi:10.1049/iet-ifs.2015.0024

43. Wang J, Chen Z, and Yuan Z. The Generation of a Hyperchaotic System Based on a Three-Dimensional Autonomous Chaotic System. Chin Phys B (2006) 15(6):1216.

44. Singh JP, and Roy BK. Simplest Hyperchaotic System with Only One Piecewise Linear Term. Electron Lett (2019) 55(7):378-80. doi:10.1049/ el.2018.8078

45. Lin H, Wang C, Yao W, and Tan Y. Chaotic Dynamics in a Neural Network with Different Types of External Stimuli. Commun Nonlinear Sci Numer Simulation (2020) 90:105390. doi:10.1016/j.cnsns.2020.105390

46. Lin H, Wang C, Hong Q, and Sun Y. A Multi-Stable Memristor and its Application in a Neural Network. IEEE Trans Circuits Syst (2020) 67(12): 3472-6. doi:10.1109/tcsii.2020.3000492

47. Cui L, Chen C, and Jin J. Dynamic Analysis and Fpga Implementation of New Chaotic Neural Network and Optimization of Traveling Salesman Problem. Complexity (2021) 2021:5521192. doi:10.1155/2021/5521192

48. Yu F, Shen H, Zhang Z, Huang Y, Cai S, and Du S. Dynamics Analysis, Hardware Realization and Engineering Applications of Novel Multi-style Attractors in a Neural Network Under Electromagnetic Radiation. Chin Phys. B (2021), 2021 in press.

49. Kwan HK. Simple Sigmoid-like Activation Function Suitable for Digital Hardware Implementation. Electron Lett (1992) 28(15):1379-80. doi:10. 1049/el:19920877

50. Liu Z, Wang Xa., Sun and Ken Lu C, and Lu K. Implementation System of Human Eye Tracking Algorithm Based on Fpga. Comp Mater Continua (2019) 58(3):653-64. doi:10.32604/cmc.2019.04597

51. Lu B, Liu F, Ge X, and Li Z. Cryptanalysis and Improvement of a Chaotic MapControl-Based and the plain Image-Related Cryptosystem. Comput Mater Continua (2019) 61(2):687-99. doi:10.32604/cmc.2019.05633

52. Liu J, Li J, Cheng J, Ma J, Sadiq N, Han B, et al. A Novel Robust Watermarking Algorithm for Encrypted Medical Image Based on Dtcwt-Dct and Chaotic Map. Comput Mater Continua (2019) 61(2):889-910. doi:10.32604/cmc.2019. 06034
53. Cheng G, Wang C, and Xu C. A Novel Hyper-Chaotic Image Encryption Scheme Based on Quantum Genetic Algorithm and Compressive Sensing. Multimedia Tools Appl (2020) 79(39-40):29 243-29. doi:10.1007/s11042-02009542-w

54. Li X, Mou J, Xiong L, Wang Z, and Xu J. Fractional-order Double-ring Erbiumdoped Fiber Laser Chaotic System and Its Application on Image Encryption. Opt Laser Tech (2021) 140:107074. doi:10.1016/j.optlastec.2021.107074

55. Zeng J, and Wang C. A Novel Hyperchaotic Image Encryption System Based on Particle Swarm Optimization Algorithm and Cellular Automata Security Commun Networks (2021) 2021:6675565. doi:10.1155/2021/ 6675565

56. Wang X, Chen S, and Zhang Y. A Chaotic Image Encryption Algorithm Based on Random Dynamic Mixing. Opt Laser Tech (2021) 138:106837. doi:10.1016/ j.optlastec.2020.106837

57. Yu F, Liu L, Qian S, Li L, Huang Y, Shi C, et al. Chaos-based Application of a Novel Multistable 5d Memristive Hyperchaotic System with Coexisting Multiple Attractors. Complexity (2020) 2020:8034196. doi:10.1155/2020/ 8034196

58. Sun J, Peng M, Liu F, and Tang C. Protecting Compressive Ghost Imaging with Hyperchaotic System and Dna Encoding. Complexity (2020) 2020:8815315. doi:10.1155/2020/8815315

59. Deng J, Zhou M, Wang C, Wang S, and Xu C. Image Segmentation Encryption Algorithm with Chaotic Sequence Generation Participated by Cipher and Multi-Feedback Loops. Multimedia Tools Appl (2021) 80: 13821-13.

60. Lin H, Wang C, Yu F, Xu C, Hong Q, Yao W, et al. “An Extremely Simple Multi-wing Chaotic System: Dynamics Analysis, Encryption Application and Hardware Implementation". IEEE Trans Industrial Electron (2020). doi:10. 1109/TIE.2020.3047012

Conflict of Interest: The authors declare that the research was conducted in the absence of any commercial or financial relationships that could be construed as a potential conflict of interest.

Copyright (c) $2021 \mathrm{Yu}$, Zhang, Shen, Huang, Cai, Jin and Du. This is an open-access article distributed under the terms of the Creative Commons Attribution License (CC $B Y)$. The use, distribution or reproduction in other forums is permitted, provided the original author(s) and the copyright owner(s) are credited and that the original publication in this journal is cited, in accordance with accepted academic practice. No use, distribution or reproduction is permitted which does not comply with these terms. 